\title{
Patterned Quantum Dot Molecule Laser Fabricated by Electron Beam Lithography and Wet Chemical Etching
}

\author{
V. B. Verma, Member, IEEE, U. Reddy, Student Member, IEEE, N. L. Dias, Student Member, IEEE, K. P. Bassett, \\ $\mathrm{X}$. Li, Senior Member, IEEE, and J. J. Coleman, Fellow, IEEE
}

\begin{abstract}
We report on the fabrication and characterization of an edge-emitting semiconductor laser with a gain medium consisting of two layers of patterned, self-aligned, vertically coupled quantum dots (QDs) using a wet-etching and regrowth technique. A threshold current density of $300 \mathrm{~A} / \mathrm{cm}^{2}$ is demonstrated at $77 \mathrm{~K}$. The presence of emission from QD excited states in both the spontaneous emission and laser spectra indicates 3-D quantum confinement in QDs fabricated using this technique.
\end{abstract}

Index Terms-Coupled quantum dots, quantum dot laser, quantum dot molecule, quantum dots.

\section{INTRODUCTION}

Q UANTUM DOT (QD) semiconductor lasers have demonstrated desirable properties when compared with traditional quantum well $(\mathrm{QW})$ based lasers such as ultralow threshold current density and reduced temperature sensitivity [1]-[6]. Typically multiple layers of QDs must be stacked in order to provide enough gain to overcome cavity losses and achieve laser threshold. Due to strain fields created by the first QD layer, the QDs in subsequently grown layers tend to selfalign with QDs in the first layer, resulting in vertical electronic coupling of the QD layers for small spacer layer thicknesses [7]-[9]. The result is effectively an array of QD "molecules," which have also been proposed as potential building blocks for solid state quantum information processing applications [10]-[12].

The most common technique for QD growth is the selfassembly technique, in which QDs form as a result of the large strain which exists between the QD material and substrate material [13]. This technique has the advantage of producing QDs of high optical quality. However, the self-assembly technique results in QDs with random positions and a broad

Manuscript received December 5, 2009; revised February 24, 2010; accepted March 7, 2010. Date of current version November 24, 2010. This work was supported in part by the Defense Advanced Research Projects Agency, under Grant No. 433 143-874a and in part by the National Science Foundation, under Grant No. 08-21979. This paper was recommended by Associate Editor A. C. Bryce.

V. B. Verma is with the National Institute of Standards and Technology, Boulder, CO 80305-3337 USA (e-mail: verma@illinois.edu).

U. Reddy, N. L. Dias, K. P. Bassett, X. Li, and J. J. Coleman are with the Department of Electrical and Computer Engineering, University of Illinois at Urbana-Champaign, Champaign, IL 61801 USA (email: cvreddy@illinois.edu; ndiasan2@illinois.edu; kpbasset@illinois.edu; xiuling@illinois.edu; jcoleman@illinois.edu).

Digital Object Identifier 10.1109/JQE.2010.2047246 size distribution which increases inhomogeneous broadening, decreases peak gain, and increases threshold current density when incorporated into a laser structure. In addition, in QD molecules fabricated using the self-assembly technique, the height and diameter of QDs have been observed to vary between layers, with the general trend being a progressive increase in diameter and thickness for QDs in subsequently grown layers [14]-[16].

Many alternative techniques have been explored to achieve better control over QD properties, such as metalorganic chemical vapor deposition (MOCVD) or molecular beam epitaxy regrowth using a patterned $\mathrm{SiO}_{2}$ growth inhibition mask [17], [18], prepatterning of InGaAs thin films [19], [20], growth in inverted pyramid shaped recesses [21], the preparation of preferential growth facets via selective area epitaxy [22], [23], manipulation of the local crystal plane step edge density [24], and etching of an existing QW or multiple QW structure [25][30]. To date there has only been one report of the use of patterned QDs in a semiconductor laser using the selective area epitaxy technique [31]. The primary challenges have been the development low-damage processing techniques which yield high QD densities as well as the development of barrier layer regrowth techniques.

Recently, we presented photoluminescence characteristics of patterned QDs fabricated using electron beam lithography and wet chemical etching which allows for the precise positioning, both spatially and spectrally, of individual QDs [32]. Here we report on the incorporation of two vertically-coupled, selfaligned layers of wet-etched patterned QDs into the active layer of an edge-emitting laser. The proposed wet etching and regrowth technique is unique in that it allows for precise engineering of the discrete energy states of each component QD of the coupled QD molecule. This can be accomplished by adjusting the indium composition and thickness of each QW before wet etching is performed using the well-established accuracy and repeatability of 2-D planar growth methods.

\section{EXPERIMENT}

The epitaxial base structure was grown by MOCVD in a Thomas Swan reactor at atmospheric pressure. The structure was grown on an $n+(100)$ GaAs substrate, and consists of a $200 \mathrm{~nm} n+\mathrm{GaAs}$ buffer layer, a $2 \mu \mathrm{m}$ n-type $\mathrm{Al}_{0.75} \mathrm{Ga}_{0.25} \mathrm{As}$ 
lower cladding, and a $100 \mathrm{~nm}$ intrinsic GaAs lower core all

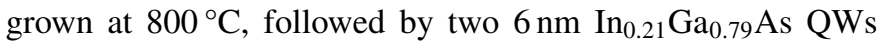
with a $6 \mathrm{~nm}$ GaAs spacer layer, and a $2 \mathrm{~nm}$ GaAs cap layer grown at $625^{\circ} \mathrm{C}$ with a V/III ratio of 50 . The spacer layer thickness is small enough to provide for electronic coupling between the QWs. The wafer was then cleaved into individual $12 \mathrm{~mm} \times 16 \mathrm{~mm}$ pieces for processing.

After a 2 min dehydration bake at $200^{\circ} \mathrm{C}$, electron beam resist consisting of a $2 \%$ solution of polymethylmethacrylate in anisole was spun on the sample to obtain a resist thickness of approximately $60 \mathrm{~nm}$. This was followed by a 2 min softbake of the resist at $200{ }^{\circ} \mathrm{C}$. The resist was exposed in a JEOL JBX6000-FS series electron beam lithography tool with a series of stripes which were $3 \mathrm{~mm}$ long and $15 \mu \mathrm{m}$ wide. Each stripe consists of a triangular array of dots on a $140 \mathrm{~nm}$ pitch, yielding a dot density of $6 \times 10^{9} \mathrm{~cm}^{-2}$. Two additional stripes were intentionally overdosed to create rectangular open areas to act as QW control devices. Alignment features were also patterned during this step using the electron beam for subsequent optical lithography steps. After exposure, the resist was developed at $10^{\circ} \mathrm{C}$ in a solution of $1: 2$ methylisobutylketone:isopropanol (IPA) for $3 \mathrm{~min}$, followed by a $30 \mathrm{~s}$ rinse in IPA and $\mathrm{N}_{2}$ dry.

After the evaporation of a $2 \mathrm{~nm}$ layer of titanium, liftoff was performed in acetone for $1 \mathrm{~h}$ followed by $15 \mathrm{~min}$ of ultrasonication to ensure complete removal of the titanium between the dots in the patterned regions. The titanium dots serve as the wet etch mask for the two underlying QWs of the base structure.

Wet etching was performed using a phosphoric acid-based etchant immediately after removal of the native surface oxide using a solution of 1:10 $\mathrm{NH}_{4} \mathrm{OH}: \mathrm{H}_{2} \mathrm{O}$. The target etch depth was $25 \mathrm{~nm}$ to ensure complete etching through both QW layers. After etching, the titanium was stripped in buffered hydrofluoric acid for $1 \mathrm{~min}$. Fig. 1 shows a scanning electron micrograph of the patterned QDs after etching. Note that the alignment features are also transferred into the sample during this wet etching step.

After a solvent clean in acetone, methanol, and IPA, the sample was cleaned in an $\mathrm{O}_{2}$ plasma at $300 \mathrm{~W}$ for $3 \mathrm{~min}$. Regrowth of the upper core of the laser structure was performed at $100 \mathrm{mbar}$ at $625^{\circ} \mathrm{C}$ in an AIX 200/4 MOCVD reactor. The upper core consists of $90 \mathrm{~nm}$ of intrinsic GaAs grown at a slow growth rate of $0.2 \mathrm{~nm} / \mathrm{s}$ and a V/III ratio of 50 . Immediately before loading the sample into the MOCVD reactor a native oxide removal step was performed in a nitrogen-purged loadlock with a 1:10 $\mathrm{NH}_{4} \mathrm{OH}: \mathrm{H}_{2} \mathrm{O}$ solution for $30 \mathrm{~s}$, followed by a $10 \mathrm{~s}$ rinse in $\mathrm{H}_{2} \mathrm{O}$ and a $20 \mathrm{~s}$ rinse in IPA.

$4 \mu \mathrm{m}$ wide ridge waveguides were then patterned over the $10 \mu \mathrm{m}$ wide stripes containing the buried QDs using standard optical lithography techniques. The ridge waveguides were etched to a depth of $125 \mathrm{~nm}$ in 1:8:80 $\mathrm{H}_{2} \mathrm{SO}_{4}: \mathrm{H}_{2} \mathrm{O}_{2}: \mathrm{H}_{2} \mathrm{O}$. The photoresist was then removed in acetone followed by a $10 \mathrm{~min}$ clean in an $\mathrm{O}_{2}$ plasma at $300 \mathrm{~W}$.

Regrowth of the upper cladding and contact layers was performed at atmospheric pressure in a Thomas Swan MOCVD reactor. A native oxide removal step was performed immediately prior to loading the sample. The final regrowth consisted of $10 \mathrm{~nm}$ of intrinsic GaAs followed by a $1.5 \mu \mathrm{m}$

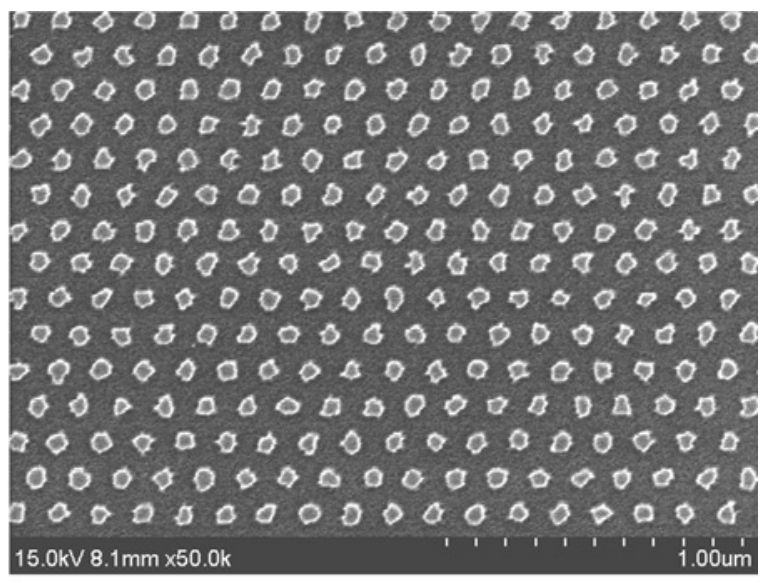

(a)

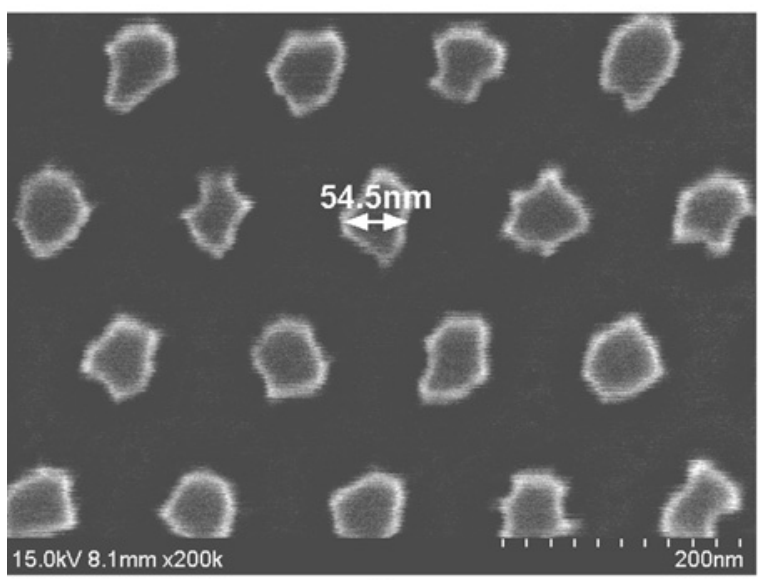

(b)

Fig. 1. SEM micrograph of the stacked QD array after wet etching and removal of the titanium etch mask at a magnification of (a) $50 \mathrm{k}$ and (b) $200 \mathrm{k}$.

p-type $\mathrm{Al}_{0.75} \mathrm{Ga}_{0.25} \mathrm{As}$ upper cladding and a $100 \mathrm{~nm}$ p+ GaAs contact layer, which completes the buried heterostructure laser shown in Fig. 2.

Following the upper cladding and contact layer regrowth, $3 \mu \mathrm{m}$ wide stripes aligned over the buried $4 \mu \mathrm{m}$ wide waveguides were etched into the $\mathrm{p}+\mathrm{GaAs}$ contact layer to reduce current spreading. $100 \mathrm{~nm}$ of $\mathrm{SiO}_{2}$ was then deposited by plasma enhanced chemical vapor deposition, followed by optical lithography to define $42 \mu \mathrm{m}$ wide vias over the $3 \mu \mathrm{m}$ wide $\mathrm{p}+\mathrm{GaAs}$ stripes. The via pattern was transferred into the underlying $\mathrm{SiO}_{2}$ using a Freon-14 reactive ion etch. p-side metals were then evaporated, followed by lapping and polishing of the substrate to a thickness of approximately $150 \mu \mathrm{m}$ to facilitate cleaving. Finally, n-side metals were deposited and annealed, and the devices were cleaved into $3 \mathrm{~mm}$ long bars for testing. The laser facets were left uncoated.

\section{Simulation}

The technique used to analyze the quantized energy states of the QDs is known as the partial function expansion method, in which the wavefunctions are expanded as the superposition 


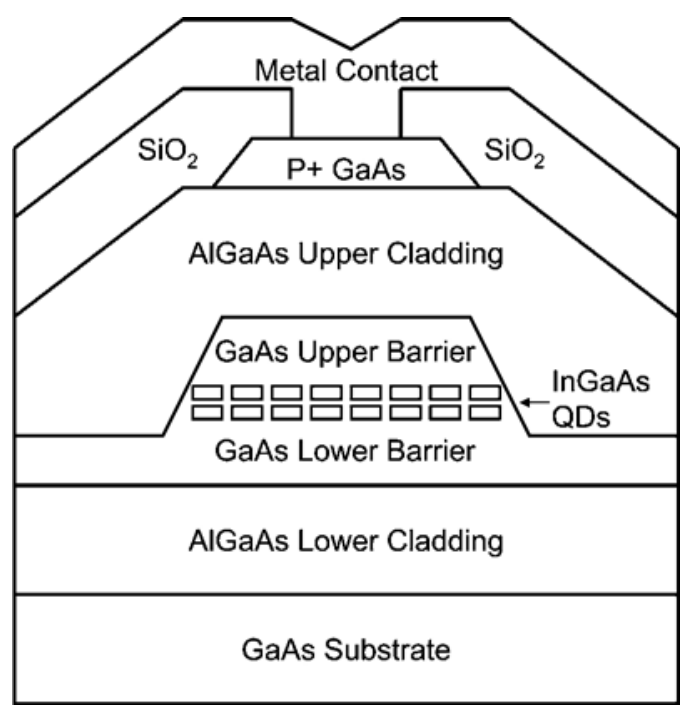

Fig. 2. Cross section diagram of the buried heterostructure laser.

of orthogonal partial functions

$$
\psi(r, \varphi, z)=\sum_{n, m, l} A_{n m l} \psi_{n m l}(r, \varphi, z)
$$

where $\psi_{n m l}$ are the orthogonal partial functions which are given by

$$
\begin{gathered}
\psi_{n m l}(r, \varphi, z)=R_{n m}(r) Z_{l}(z) \varphi_{m}(\varphi) \\
\varphi_{m}(\varphi)=\frac{1}{\sqrt{2 \pi}} e^{i m \varphi} \\
R_{n m}(r)=\frac{\sqrt{2}}{\rho_{0} J_{m+1}\left(k_{n} \rho_{0}\right)} J_{m}\left(k_{n} r\right) \\
Z_{l}(z)=\sqrt{\frac{2}{H}} \sin l \pi\left(\frac{1}{2}-\frac{z}{H}\right) .
\end{gathered}
$$

The QD is assumed to be embedded in a large boundary cylinder of height $H$ and radius $\rho_{0}$. The wavefunction is zero on the boundaries of this cylinder by design of the partial functions. Inside the QD, the particle has effective mass $m^{*}(r, z)=m_{w}$, and the potential has the value $V(r, z)=0$. In the matrix material occupying the region between the QD and boundary cylinder, the particle has effective mass $m^{*}(r, z)=m_{b}$ and the potential has the value $V(r, z)=V_{0}$. Using (1) in the Schrödinger equation

$$
-\frac{\hbar^{2}}{2} \nabla \cdot \frac{1}{m^{*}(r, z)} \nabla \psi+V(r, z) \psi=E \psi
$$

yields an equation of the form

$$
\begin{aligned}
& -\frac{\hbar^{2}}{2} \nabla \cdot \frac{1}{m^{*}(r, z)} \nabla \sum_{n, m, l} A_{n m l} \psi_{n m l}+V(r, z) \sum_{n, m, l} A_{n m l} \psi_{n m l} \\
& =E \sum_{n, m, l} A_{n m l} \psi_{n m l} .
\end{aligned}
$$

Multiplying both sides of the equation by $\psi_{n^{\prime} m^{\prime} l^{\prime}}^{*}$ and integrating over the 3-D boundary cylinder yields a matrix equation of the form

$$
\sum_{n, m, l} A_{n m l} M_{n m l n^{\prime} m^{\prime} l^{\prime}}=E A_{n^{\prime} m^{\prime} l^{\prime}}
$$

Spatial variations of the effective mass and potential are taken into account by dividing the integration into parts in which the effective mass and potential are constant. Further details have been outlined in [33]. For simplicity we assumed perfectly cylindrical QD shape for our calculations.

Strain-induced band edge shifts were calculated using commercial 3-D finite element analysis software (ABAQUS). Fig. 3 shows the finite element model used for the analysis. The model consists of two $6 \mathrm{~nm}$ thick, $55 \mathrm{~nm}$ diameter cylindrical QDs with a $6 \mathrm{~nm}$ GaAs spacer layer embedded in $50 \mathrm{~nm}$ thick GaAs layers. The central QD/spacer region and the upper and lower GaAs barrier layers were each created as separate parts. A tied contact condition was used between the barrier layers and the central QD/spacer layer to model rigid contact between the three sections of the model. A 6-node linear triangular prism element was used to create the mesh, with a higher mesh density used in the QD/spacer region of the model. All displacement components for nodes on the base of the model were constrained, while for the vertical faces the normal component of motion was constrained to model the periodicity of the structure. Orthotropic elasticity tensors were used for both the GaAs and $\operatorname{In}_{0.21} \mathrm{Ga}_{0.79} \mathrm{As}$ materials. The strain induced due to lattice mismatch was simulated by assigning a thermal expansion coefficient of $\alpha_{T}=\left(a-a_{0}\right) / a=0.015$ to the $\operatorname{In}_{0.21} \mathrm{Ga}_{0.79}$ As material and raising the model temperature by $1 \mathrm{~K}$, where $a_{0}$ is the lattice constant of the GaAs substrate and $a$ is the lattice constant of $\mathrm{In}_{0.21} \mathrm{Ga}_{0.79}$ As. This effectively results in an expansion of the $\mathrm{In}_{0.21} \mathrm{Ga}_{0.79} \mathrm{As}$ material in the simulation and contributes to the force vectors in the finite element equations. The band-edge shifts were computed from the components of the strain tensor produced by the simulation using the following equations:

$$
\begin{gathered}
\Delta E_{c}=a_{c}\left(\varepsilon_{x x}+\varepsilon_{y y}+\varepsilon_{z z}\right) \\
\Delta E_{H H}=-P-\operatorname{sgn}(Q) \sqrt{|Q|^{2}+|R|^{2}+|S|^{2}} \\
P=-a_{v}\left(\varepsilon_{x x}+\varepsilon_{y y}+\varepsilon_{z z}\right) \\
Q=-\frac{b}{2}\left(\varepsilon_{x x}+\varepsilon_{y y}-2 \varepsilon_{z z}\right) \\
R=\frac{\sqrt{3}}{2} b\left(\varepsilon_{x x}-\varepsilon_{y y}\right)-i d \varepsilon_{x y} \\
S=-d\left(\varepsilon_{x z}-i \varepsilon_{y z}\right)
\end{gathered}
$$

where $a_{c}, a_{v}, b$, and $d$ are the deformation potentials for $\mathrm{In}_{0.21} \mathrm{Ga}_{0.79}$ As.

The conduction band edge shift was found to be $\Delta E_{c}=123 \mathrm{meV}$ at the center of the QD. The $123 \mathrm{meV}$ shift represents an increase of $14 \mathrm{meV}$ relative to the conduction band shift of $109 \mathrm{meV}$ in a double $\mathrm{In}_{0.21} \mathrm{Ga}_{0.79} \mathrm{As} / \mathrm{GaAs}$ QW. The computed shift in the $\mathrm{HH}$ band edge was $\Delta E_{H H}=15.5 \mathrm{meV}$ at the center of the QD. This shift represents a decrease of $16.5 \mathrm{meV}$ relative to a double QW for which $\Delta E_{H H}=32 \mathrm{meV}$. Thus, the expected blueshift in emission wavelength of the stacked QDs relative to a stacked QW due strain effects alone amounts to approximately $30.5 \mathrm{meV}$ or 


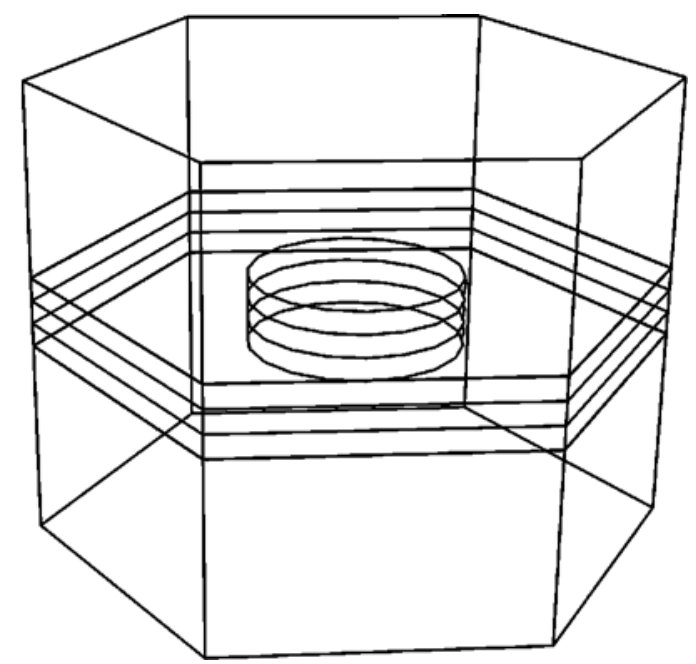

Fig. 3. Finite element model of a quantum dot molecule used for modeling the strain-induced shifts in the conduction and valence band edges.

$20 \mathrm{~nm}$. This shift can be attributed to an increase in hydrostatic strain or a decrease in QD volume caused by the GaAs barrier material acting on the vertical sidewalls of the QD.

In order to simplify the calculation of the electron and heavy hole confined energies, we assumed that the band edge energies were constant throughout the QD volume and equal to the value at the center of the QD. This is a good approximation considering most of the variation occurs very close to the edges of the QD and the variations are much smaller than the potential barrier height between $\operatorname{In}_{0.21} \mathrm{Ga}_{0.79} \mathrm{As}$ and GaAs. It was found that in vertically coupled QDs with $55 \mathrm{~nm}$ diameter, the increase in the electron ground state energy relative to a stacked QW is only $0.8 \mathrm{meV}$, while the increase in the heavy hole ground state energy is approximately $0.2 \mathrm{meV}$. Thus, the blueshift due to quantum size effects is negligible compared to the blueshift due to strain-induced shifts in the band edges for QDs of this diameter and composition.

From these calculations of the electron and hole confined state energies, we can conclude that at a diameter of $55 \mathrm{~nm}$, the small variations in the sizes and shapes of the QDs observed in Fig. 1 should lead to correspondingly small variations in emission energies on the order of $1 \mathrm{meV}$. Thus, inhomogenous broadening due to size and shape variation should be minimal in QDs of this diameter which is significantly larger than the typical diameter of self-assembled QDs.

\section{RESULTS}

The QD lasers were tested under pulsed operation in a liquid nitrogen cryostat at $77 \mathrm{~K}$ with a $2 \mu$ s pulse width and $0.3 \%$ duty cycle. The laser emission was focused into an $\mathrm{f} / 101 \mathrm{~m}$ monochromator and detected using a liquid nitrogen-cooled Ge detector. Fig. 4 shows amplified spontaneous emission spectra collected from the laser facet at a current of $10 \mathrm{~mA}$ from a QD laser and a QW laser. The band edge of the QD emission is blueshifted relative to that of the QW emission by approximately $20 \mathrm{~nm}$ which is in good agreement with the theoretical predictions of the strain-induced blueshift. Note that unlike the spontaneous emission from typical self-

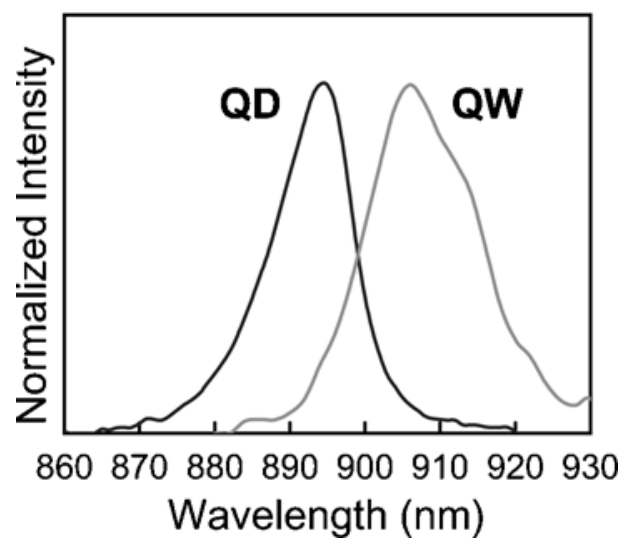

Fig. 4. Comparison of QD and QW amplified spontaneous emission spectra at a current of $10 \mathrm{~mA}$.

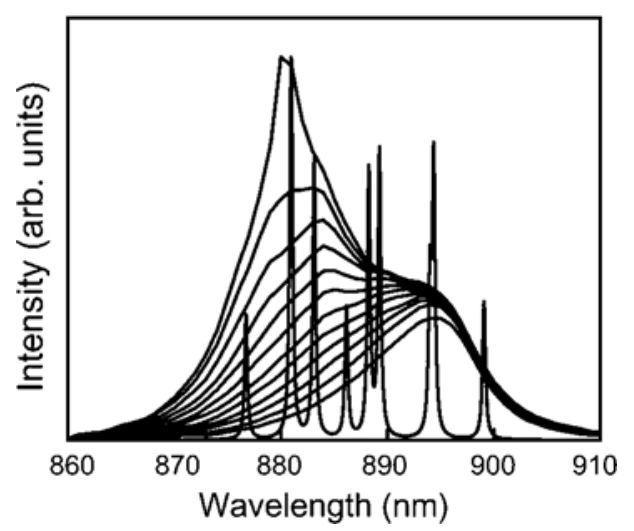

Fig. 5. Evolution of the amplified spontaneous emission spectrum of the QD laser below threshold between currents of $10 \mathrm{~mA}$ and $32 \mathrm{~mA}$ in $2 \mathrm{~mA}$ steps. The computed joint density of states is also shown with $0.5 \mathrm{meV}$ broadening.

assembled QDs, the emission linewidth of the patterned QDs is comparable to that of the reference QW. This is to be expected based on the relatively large diameter of the patterned QDs compared to self-assembled QDs, which results in reduced inhomogeneous broadening due to size and shape variations. Fig. 5 shows the evolution of the amplified spontaneous emission spectrum for a QD laser with increasing current along with the theoretically predicted joint density of states taking into account the magnitude of the dipole matrix element for each transition. The peak at $880 \mathrm{~nm}$ is the result of three nearly degenerate transitions resulting from the confined electron and heavy hole states with quantum numbers $(n, m, l)=(2, \pm 3$, $\left.1^{-}\right)$, and $\left(3,0,1^{+}\right)$, where $1^{-}$and $1^{+}$represent the lowest energy bonding and antibonding states for the coupled QDs along the growth axis, respectively. The states with quantum numbers $\left(2, \pm 3,1^{-}\right)$are exactly degenerate in energy, while the $\left(3,0,1^{+}\right)$state is separated from these degenerate states by only $0.1 \mathrm{meV}$. The clear presence of excited states in the amplified spontaneous emission spectra close to threshold provides evidence for the 3-D confinement of carriers in these QDs.

Fig. 6 shows the amplified spontaneous emission linewidth as a function of current, while Fig. 7 shows the integrated power as a function of current. The drastic decrease in 


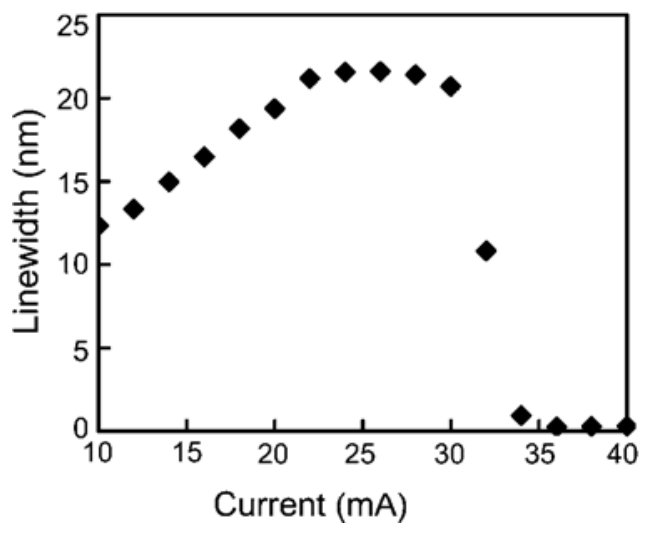

Fig. 6. Emission linewidth as a function of current.

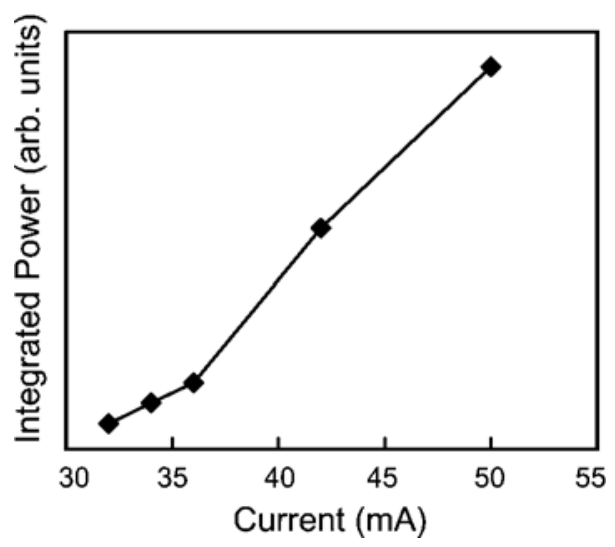

Fig. 7. Integrated power as a function of current.

linewidth and increase in integrated power above $36 \mathrm{~mA}$ determine the onset of lasing, corresponding to a threshold current density of $300 \mathrm{~A} / \mathrm{cm}^{2}$. This value is higher than the threshold for the double QW control device which demonstrated a threshold current of $20 \mathrm{~mA}$ corresponding to a threshold current density of $167 \mathrm{~A} / \mathrm{cm}^{2}$.

The threshold current density is given by

$$
J_{\mathrm{th}}=\frac{q d n_{\mathrm{th}}}{\tau_{e}\left(n_{\mathrm{th}}\right)}
$$

where $d$ is the QW thickness, $\tau_{e}$ is the carrier lifetime, and $n_{\text {th }}$ is the sum of the transparency carrier density and the carrier density required to overcome the cavity losses

$$
n_{\mathrm{th}}=n_{\mathrm{tr}}+\frac{\alpha_{i}+\alpha_{m}}{\Gamma g_{0}}
$$

where $\alpha_{i}$ is the intrinsic cavity loss, $\alpha_{m}$ is the mirror loss, $\Gamma$ is the optical confinement factor, and $g_{0}$ is the differential gain. The optical confinement factor is the product of an in-plane confinement factor $\Gamma_{x y}$ and a transverse confinement factor $\Gamma_{z}$ along the growth direction. For a QW, $\Gamma_{x y}=1$, while for a QD array with $55 \mathrm{~nm}$ diameter QDs on a $140 \mathrm{~nm}$ pitch, $\Gamma_{x y}=1 / 5$. $\Gamma_{z}$ is identical for both the QDs and the QWs. The ratio of $\mathrm{QD}$ and QW threshold carrier densities is then given by

$$
\frac{J_{\mathrm{th}}^{P Q D}}{J_{\mathrm{th}}^{Q W}}=\frac{n_{\mathrm{tr}}^{P Q D}+5\left(\alpha_{i}+\alpha_{m}\right) /\left(\Gamma_{z} g_{0}^{P Q D}\right)}{n_{\mathrm{tr}}^{Q W}+\left(\alpha_{i}+\alpha_{m}\right) /\left(\Gamma_{z} g_{0}^{Q W}\right)} \cdot \frac{\tau_{e}^{Q W}}{\tau_{e}^{P Q D}} .
$$

In an ideal QD laser one would expect both the transparency and threshold carrier densities to be lower than in a comparable QW laser due to the reduced active region volume and the higher differential gain resulting from the discrete density of states. There are a number of nonidealities in the patterned QD array which could lead to a higher threshold carrier density. First, the QD laser lases on an excited state approximately $20 \mathrm{~nm}$ from the band edge. In order to reach threshold, all of the low energy states must first be populated, which will significantly increase $J_{\mathrm{th}}^{P Q D}$. Second, inhomogeneous broadening will cause a reduction in $g_{0}^{P Q D}$ which in turn will result in an increase in the component of the carrier density necessary to overcome the cavity losses. Third, the confinement factor is a factor of 5 lower than in the QW, which will cause an additional increase in the cavity loss component of the carrier density. Finally, it is possible that the lifetime of carriers in the QDs is reduced due to the presence of nonradiative recombination centers introduced during the processing and regrowth steps, resulting in an increase of the ratio of the carrier lifetimes appearing in (17).

Typically, the transparency carrier density can be extracted experimentally by cleaving devices of different lengths. Plotting the inverse external quantum efficiency as a function of length yields the internal loss $\alpha_{i}$, and the mirror loss is given by $\alpha_{m}=1 / L \ln (1 / R)$ where $R$ is the facet reflectivity. Plotting the threshold modal gain, which is equal to the total cavity loss $\alpha_{i}+\alpha_{m}$, as a function of threshold current density and fitting to the empirical equation

$$
g=g^{\mathrm{sat}}\left[1-\exp \left(-\gamma\left(J-J_{0}\right) / J_{0}\right)\right]
$$

yields the QD saturated modal gain $g^{\text {sat }}$ and the transparency carrier density $J_{0}$ [34]. However, because the patterned QD devices are fabricated by electron beam lithography, it is impractical to fabricate enough devices of various lengths to obtain a meaningful statistical average of these parameters, and at present we must rely on the qualitative arguments given above to explain the relative increase in the observed threshold carrier density of the patterned QD lasers.

Fig. 8 shows the QD and QW normalized laser emission spectra with both devices biased just above threshold. The QD emission spectrum was obtained at a resolution of $0.5 \mathrm{~nm}$, while the QW spectral resolution was set to $0.05 \mathrm{~nm}$. Note that although the spontaneous emission band edges are separated by approximately $20 \mathrm{~nm}(30 \mathrm{meV})$ at low currents as shown in Fig. 4, the QD device lases on an excited state at $880 \mathrm{~nm}$ where the density of states is largest as shown in Fig. 5, resulting in a $30 \mathrm{~nm}(46 \mathrm{meV})$ blueshift of the QD laser emission relative to the QW laser emission.

Fig. 9 presents the evolution of the QD laser emission spectrum as a function of current between currents of $50 \mathrm{~mA}$ and $300 \mathrm{~mA}$. As the current is increased multiple excited states appear in the spectra due to gain saturation of the low energy states. These states were not found to be present in the QW laser spectra at high currents. Furthermore, the small spacing between these states in energy is consistent with that expected for large QDs. We take these observations to be direct evidence of 3-D quantum confinement. 


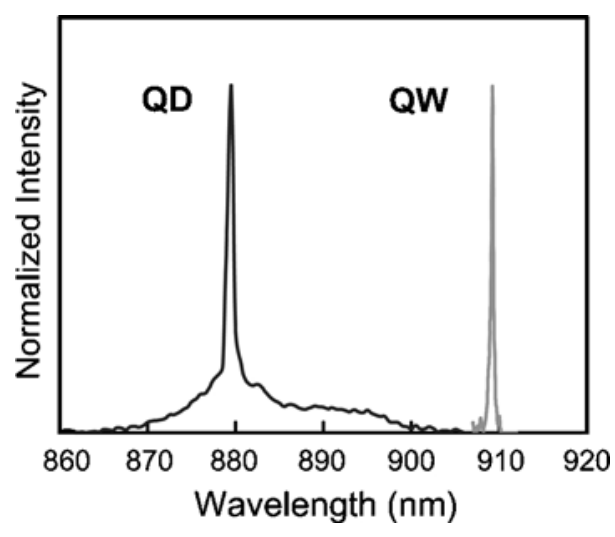

Fig. 8. Comparison of QD and QW lasers with each laser biased just above threshold.

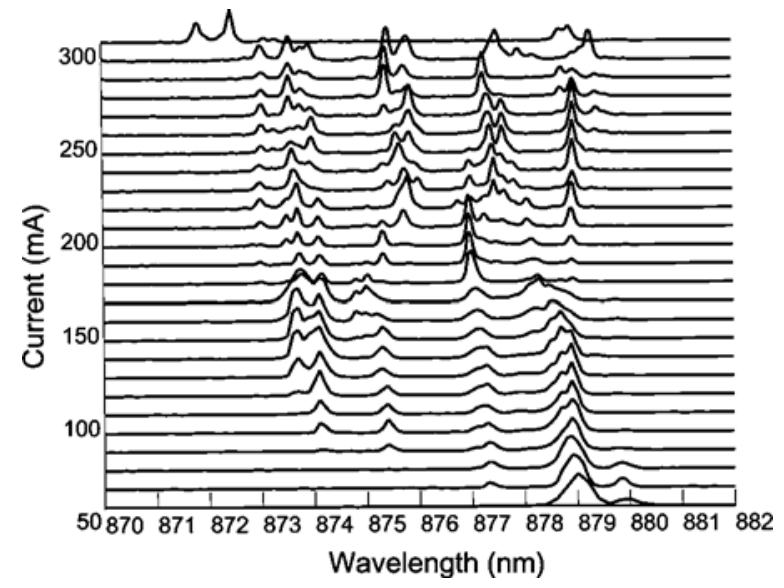

Fig. 9. Evolution of QD emission spectrum as a function of current above threshold.

\section{CONCLUSION}

We have demonstrated for the first time the use of electronically coupled patterned QDs fabricated using a wet etching and regrowth technique as the gain medium of a photonic device. This technique provides the unique ability to control both the spatial and spectral properties of QDs, while maintaining the high optical quality of the material necessary for photonics applications. We expect this technique to find applications in all types of photonic devices from edge-emitting lasers to vertical cavity surface emitting lasers, in addition to devices requiring the precise control of the position and emission wavelength of single QDs or QD "molecules" consisting of two vertically coupled QDs.

We note that the presented fabrication process has certain limitations imposed by the fact that one must begin with 2-D QWs. The indium composition and thickness of these QWs, and hence, the emission wavelength, are limited by the Mathews-Blakeslee critical layer thickness. Thus, long wavelength emission, which is easily achieved with selfassembled QDs, would in principle be difficult to achieve using the wet-etching approach. However, this approach may be useful for certain shorter wavelength applications which are not within reach of typical self-assembled QDs.

Finally, the presented fabrication process also provides for the possibility of realizing "designer" QD molecules in which the properties of each of the two coupled QDs can be controlled independently by adjusting the indium composition or thickness of each QW layer. This flexibility may prove useful for many proposed implementations of quantum information processing using QD molecules.

\section{REFERENCES}

[1] H. Shimizu, S. Saravanan, J. Yoshida, S. Ibe, and N. Yokouchi, "InAs quantum dot lasers with extremely low threshold current density (7 A/cm²/layer)," Jpn. J. Appl. Phys., vol. 44, no. 35, pp. L1103-L1104, 2005.

[2] G. Park, O. B. Shchekin, S. Csutak, D. L. Huffaker, and D. G. Deppe, "Room-temperature continuous-wave operation of a singlelayered $1.3 \mu \mathrm{m}$ quantum dot laser," Appl. Phys. Lett., vol. 75, no. 21, pp. 3267-3269, 1999.

[3] I. I. Novikov, M. V. Maksimov, Y. M. Shernyakov, N. Y. Gordeev, A. R. Kovsh, A. E. Zhukov, S. S. Mikhrin, N. A. Maleev, A. P. Vasil'ev, V. M. Ustinov, Z. I. Alferov, N. N. Ledentsov, and D. Bimberg, "Temperature characteristics of low-threshold high-efficiency quantum-dot lasers with emission wavelength from 1.25 to $1.29 \mu \mathrm{m}$," Semiconductors, vol. 37, no. 10 , pp. 1239-1242, 2003.

[4] D. L. Huffaker, G. Park, Z. Zou, O. B. Shchekin, and D. G. Deppe, "1.3 $\mu \mathrm{m}$ room-temperature GaAs-based quantum-dot laser," Appl. Phys. Lett, vol. 73, no. 18, pp. 2564-2566, 1998.

[5] G. T. Liu, A. Stintz, H. Li, K. J. Malloy, and L. F. Lester, "Extremely low room-temperature threshold current density diode lasers using InAs dots in $\operatorname{In}_{0.15} \mathrm{Ga}_{0.85}$ as quantum wells," Electron. Lett., vol. 35, no. 14, p. $1163,1999$.

[6] M. V. Maximov, I. V. Kochnev, Y. M. Shernyakov, S. V. Zaitsev, N. Y. Gordeev, A. F. Tsatsul'nikov, A. V. Sakharov, I. L. Krestnikov, P. S. Kop'ev, Z. I. Alferov, N. N. Ledentsov, D. Bimberg, A. O. Kosogov, P. Werner, and U. Gösele, "InGaAs/GaAs quantum dot lasers with ultrahigh characteristic temperature $\left(\mathrm{T}_{0}=385 \mathrm{~K}\right)$ grown by metal organic chemical vapour deposition," Jpn. J. Appl. Phys., vol. 36, no. 6b, pp. 4221-4223, 1997.

[7] S. Sanguinetti, M. Padovani, M. Fuioli, E. Grilli, M. Guzzi, A. Vinattieri, M. Colocci, P. Frigeri, S. Fanchi, L. Lazzarini, and G. Salviati, "Study of $\mathrm{GaAs}$ spacer layers in InAs/GaAs vertically aligned quantum dot structures," Thin Solid Films, vol. 380, nos. 1-2, pp. 224-226, 2000.

[8] H. S. Kim, J. H. Suh, C. G. Park, S. J. Lee, S. K. Noh, J. D. Song, Y. J. Park, W. J. Choi, and J. I. Lee, "Effects of the thickness of GaAs spacer layers on the structure of multilayer stacked InAs quantum dots," J. Crystal Growth, vol. 311, no. 2, pp. 258-262, 2009.

[9] T. Benabbas, Y. Androussi, and A. Lefebvre, "A finite-element study of strain fields in vertically aligned InAs islands in GaAs," J. Appl. Phys., vol. 86, no. 4, pp. 1945-1950, 1999.

[10] K. C. Wijesundara, M. Garrido, S. Ramanathan, E. A. Stinaff, M. Scheibner, A. S. Bracker, and D. Gammon, "Electric field tunable exchange interaction in InAs/GaAs couopled quantum dots," in Proc. Mater. Res. Soc. Symp., vol. 1117E. 2009, pp. 1117-J04-08.R1.

[11] E. A. Stinaff, M. Scheibner, A. S. Bracker, I. V. Ponomarev, V. L. Korenev, M. E. Ware, M. F. Doty, T. L. Reinecke, and D. Gammon, "Optical signatures of coupled quantum dots," Science, vol. 311, no. 5761, pp. 636-639, 2006.

[12] G. D. Sanders, K. W. Kim, and W. C. Holton, "Scalable solid-state quantum computer based on quantum dot pillar structures," Phys. Rev. $B$, vol. 61 , no. 11 , pp. 7526-7535, 2000.

[13] F. Tinjod and H. Mariette, "Self-assembled quantum dot formation induced by surface energy change of a strained 2-D layer," Phys. Stat. Sol. (b), vol. 241, no. 3, pp. 550-557, 2004.

[14] Q. Xie, A. Madhukar, P. Chen, and N. P. Kobayashi, "Vertically selforganized InAs quantum box islands on GaAs (100)," Phys. Rev. Lett., vol. 75 , no. 13, pp. 2542-2545, 1995.

[15] H. Heidemeyer, S. Kiravittaya, C. Müller, N. Y. Jin-Phillipp, and O. G. Schmidt, "Closely stacked InAs/GaAs quantum dots grown at low growth rate," Appl. Phys. Lett., vol. 80, no. 9, pp. 1544-1546, 2002.

[16] D. Pal, E. Towe, and S. Chen, "Structural characterization of InAs/GaAs quantum-dot nanostructures," Appl. Phys. Lett., vol. 78, no. 26, pp. 4133-4135, 2001.

[17] Y. Nagamune, M. Nishioka, S. Tsukamoto, and Y. Arakawa, "GaAs quantum dots with lateral dimension of $25 \mathrm{~nm}$ fabricated by selective metalorganic chemical vapor deposition growth," Appl. Phys. Lett., vol. 64, no. 19, pp. 2495-2497, 1994. 
[18] S. C. Lee, A. Stintz, and S. R. J. Brueck, "Nanoscale limited area growth of InAs islands on GaAs (001) by molecular beam epitaxy," J. Appl. Phys., vol. 91, no. 5, pp. 3282-3288, 2002.

[19] T. S. Yeoh, C. P. Liu, R. B. Swint, A. E. Huber, S. D. Roh, C. Y. Woo, K. E. Lee, and J. J. Coleman, "Epitaxy of InAs quantum dots on self-organized 2-D InAs islands by atmospheric pressure metalorganic chemical vapor deposition," Appl. Phys. Lett., vol. 79, no. 2, pp. $221-$ 223, 2001

[20] T. S. Yeoh, R. B. Swint, A. Gaur, V. C. Elarde, and J. J. Coleman, "Selective growth of InAs quantum dots by metalorganic chemical vapor deposition," IEEE J. Select. Topics Quantum Electron., vol. 8, no. 4, pp. 833-838, Jul.-Aug. 2002.

[21] S. Watanabe, E. Pelucchi, B. Dwir, M. H. Baier, K. Leifer, and E. Kapon, "Dense uniform arrays of site-controlled quantum dots grown in inverted pyramids," Appl. Phys. Lett., vol. 84, no. 15, pp. 2907-2909, 2004.

[22] C.-K. Hahn, J. Motohisa, and T. Fukui, "Position and number control of self-assembled InAs quantum dots by selective area metalorganic vaporphase epitaxy," J. Crystal Growth, vol. 221, nos. 1-4, pp. 599-604, 2000.

[23] C.-K. Hahn, J. Motohisa, and T. Fukui, "Formation of single and double self-organized InAs quantum dot by selective area metal-organic vapor phase epitaxy," Appl. Phys. Lett., vol. 76, no. 26, pp. 3947-3949, 2000.

[24] T. Ishikawa, S. Kohmoto, and K. Asakawa, "Site control of selforganized InAs dots on GaAs substrates by in situ electron-beam lithography and molecular-beam epitaxy," Appl. Phys. Lett., vol. 73, no. 12, pp. 1712-1714, 1998.

[25] A. Scherer, and H. G. Craighead, "Fabrication of small laterally patterned multiple quantum wells," Appl. Phys. Lett., vol. 49, no. 19, pp. 1284-1286, 1986

[26] E. M. Clausen, Jr., H. G. Craighead, J. M. Worlock, J. P. Harbison, L. M. Schiavone, L. Florez, and B. Van der Gaag, "Determination of nonradiative surface layer thickness in quantum dots etched from single quantum well GaAs/AlGaAs," Appl. Phys. Lett., vol. 55, no. 14, pp. $1427-1429,1989$

[27] A. Forschel, H. Leier, B. E. Maile, and R. Germann, "Fabrication and optical spectroscopy of ultrasmall III-V compound semiconductor structures," Advances Solid State Phys., vol. 28, pp. 99-119, 1988.

[28] P. M. Petroff, A. C. Gossard, R. A. Logan, and W. Wiegmann, "Toward quantum well wires: Fabrication and optical properties," Appl. Phys. Lett., vol. 41, no. 7, pp. 635-638, 1982.

[29] A. Forchel, R. Steffen, T. Koch, M. Michel, M. Albrecht, and T. L. Reinecke, "Optical studies of free-standing single InGaAs/GaAs quantum dots," Semicond. Sci. Technol., vol. 11, no. 11S, pp. 1529$1533,1996$.

[30] R. Steffen, T. Koch, J. Oshinowo, F. Faller, and A. Forchel, "Photoluminescence study of deep etched InGaAs/GaAs quantum wires and dots defined by low-voltage electron beam lithography," Appl. Phys. Lett., vol. 68, no. 2, pp. 223-225, 1996.

[31] V. C. Elarde, R. Rangarajan, J. J. Borchardt, and J. J. Coleman, "Roomtemperature operation of patterned quantum-dot lasers fabricated by electron beam lithography and selective area metal-organic chemical vapor deposition," IEEE Photon. Technol. Lett., vol. 17, no. 5, pp. 935937, May 2005.

[32] V. B. Verma and J. J. Coleman, "High density patterned quantum dot arrays fabricated by electron beam lithography and wet chemical etching," Appl. Phys. Lett., vol. 93, no. 11, p. 111117, 2008.

[33] J. Lee, W.-C. Chou, C.-S. Yang, and G. J. Jan, "Eigen-energies and eigen-functions of symmetroidal quantum dots," Chin. J. Phys., vol. 42, no. 1, pp. 102-115, 2004.

[34] A. E. Zhukov, A. R. Kovsh, V. M. Ustinov, A. Y. Egorov, N. N Ledentsov, A. F. Tsatsul'nikov, M. V. Maximov, Y. M. Shernyakov, V. I. Kopchatov, A. V. Lunev, P. S. Kop'ev, D. Bimberg, and Z. I. Alferov, "Gain characteristics of quantum dot injection lasers," Semicond. Sci. Technol., vol. 14, no. 1, pp. 118-123, 1999.

V. B. Verma (S'06-M'09) received the B.S. degree in computer engineering, the M.S. degree in electrical engineering, and the Ph.D. degree in electrical engineering, all from the University of Illinois at Urbana-Champaign, Champaign, in 2004, 2005, and 2009, respectively.

Currently, he is with the National Institute of Standards and Technology, Boulder, CO. His research interests include nanoscale processing techniques, crystal growth of III-V materials, high power, narrow linewidth distributed Bragg reflector lasers, and the use of patterned quantum dots for applications in quantum optics and quantum information processing.
U. Reddy (S'09) received the B.Eng. degree in electrical engineering from the University of Illinois at Urbana-Champaign, Champaign, in 2005, and the M.S. degree from the same university in 2007 . He is currently pursuing the $\mathrm{Ph}$.D. degree in electrical engineering from the University of Illinois at Urbana-Champaign under the guidance of Prof. James Coleman.

His current research interests include high-power, narrow-linewidth semiconductor lasers.

N. L. Dias (S'09) received the B.Eng. degree from the Karnataka Regional Engineering College, Mangalore, India, and the M.S. degree in electrical engineering from the University of Wisconsin-Madison, Madison. Since 2007, he has been pursuing the Ph.D. degree from the University of Illinois at Urbana-Champaign, Champaign.

His current research interests include the design and fabrication of patterned quantum dot optoelectronic devices and narrow linewidth distributed Bragg grating lasers.

K. P. Bassett received the B.S. degree in electrical engineering from the University of Illinois at Urbana-Champaign, Champaign, in 2007. He is pursuing the M.S. and Ph.D. degrees in electrical engineering from the University of Illinois at Urbana-Champaign.

He is currently a Research Assistant with the Micro and Nano Technology Laboratory, University of Illinois at Urbana-Champaign.

X. Li (SM'08) received the Ph.D. degree from the University of California at Los Angeles, Los Angeles.

After working with a startup company for six years, she became a Faculty Member with the University of Illinois at Urbana-Champaign, Champaign, in 2007. She is currently an Assistant Professor in the Department of Electrical and Computer Engineering in the same university. She is also an Affiliate Faculty Member of the Department of Materials Science and Engineering, and the Beckman Institute for Advanced Science and Technology. Her current research interests include the area of nanostructured semiconductor materials and devices.

Dr. Li has won the National Science Foundation CAREER Award in 2008, and Defense Advanced Research Projects Agency Young Faculty Award in 2009. She has served on the semiconductor laser subcommittee for the IEEE Photonic Society for the past two years.

J. J. Coleman (S'73-M'76-SM'80-F'92) received the B.S., M.S., and Ph.D degrees in electrical engineering from the University of Illinois at UrbanaChampaign, Champaign.

He was with Bell Laboratories, Murray Hill, NJ, where he contributed to the development of long wavelength $1.3 \mathrm{~mm}$ InGaAsP CW room temperature diode lasers grown by liquid phase epitaxy.

At Rockwell International, Anaheim, CA, he studied metalorganic chemical vapor deposition-grown low-threshold single mode AlGaAs-GaAs double heterostructure and quantum well heterostructure laser devices. He presently holds the Intel Alumni Endowed Chair in the Department of Electrical and Computer Engineering, University of Illinois at Urbana-Champaign. He and his students were the first group to define experimentally the ranges of wavelength, threshold current density, and reliability of $980 \mathrm{~nm}$ strained-layer InGaAs lasers. They are presently involved in developing high performance narrow linewidth distributed Bragg reflector lasers, integrable lasers and other photonic devices by selective-area epitaxy, and the growth processes for quantum dot and quantum wire lasers. He has published more than 400 papers in technical journals and thirteen book chapters. He has seven U.S. patents and has given more than 80 invited presentations.

Dr. Coleman has served as an Associate Editor of the IEEE PHOTONICS TECHNOLOGY LETTERS and as a Guest Editor for two Special Issues of the IEEE JOURNAL OF QUANTUM ELECTRONICS and three other Special Issues of the IEEE JOURNAL OF SELECTED TOPICS IN QUANTUM ELECTRONICS. He is the Former Chair of the IEEE Photonics Society, the Semiconductor Lasers Technical Committee, and served as the Photonics Society Vice-President for Publications. He is currently the President of the Photonics Society. He has won the IEEE David Sarnoff Award, the IEEE Photonics Society Distinguished Service Award, the Optical Society of America Nick Holonyak, Jr. Award, the International Symposium on Compound Semiconductors Heinrich Welker Award, the IEEE Photonics Society William Streifer Scientific Achievement Award, and was an IEEE Photonics Society Distinguished Lecturer. He is a Fellow of the Optical Society of America, the American Physical Society, and the American Association for the Advancement of Science. 